Bundesgesundheitsbl - Gesundheitsforsch Gesundheitsschutz 2004 · 47:647-652 DOI 10.1007/s00103-004-0868-6 ๑) Springer-Verlag 2004

H. Schmitz · C. Drosten

Bernhard-Nocht-Institut für Tropenmedizin, Hamburg

\title{
Die Bedeutung von Coronaviren
}

\author{
Das Beispiel SARS
}

islang waren Coronaviren bei Menschen vorwiegend als Erreger von grippalen Infekten und als harmlose Darmbewohner bekannt. Die Coronavirus-Epidemie, die zum Jahresende 2002 von Südchina ausging, hat gezeigt, dass es hochpathogene Coronaviren nicht nur beim Tier, sondern auch bei Menschen gibt, die sich schnell durch Interkontinentalflüge weltweit ausbreiten können.

\section{Coronaviren}

Coronaviren sind 1937 zuerst bei Vögeln und erst ca. 30 Jahre später auch beim Menschen [1] nachgewiesen worden. Die menschenpathogenen Stämme sollen nach älteren Studien für 30\% aller leichten grippeartigen Erkrankungen in der Bevölkerung verantwortlich sein [2]. Im Vergleich zu RS- (Respiratory-Syncytial) Virusinfektionen treten Coronavirus-Infektionen beim Menschen jedenfalls selten auf [3]. Bei immunsupprimierten Personen ließen sich diese Viren elektronenoptisch häufig im Stuhl nachweisen, ohne dass sie in Verbindung mit ernsteren Symptomen gebracht werden konnten [4]. Deshalb wurde dieser Virusfamilie zumindest im humanmedizinischen Bereich bis vor kurzem keine allzu große Beachtung geschenkt. Im veterinärmedizinischen Bereich spielen Coronaviren eine große Rolle. Hier rufen sie bei verschiedenen Säugern (Serogruppen 1 und 2) schwere Gastroenteritiden/Diarrhöen (Schwein/Rind), Hepatitiden (Maus) und Peritonitiden (Katzen) hervor. Bei Vögeln lösen Coronaviren der Gruppe 3 Bronchitiden aus. Es besteht also eine hohe Affinität der Coronaviren zu Epithelzellen des Respirations- und des Gastrointestinaltraktes. Einige Stämme befallen auch Leber- und Nervenzellen. Vor einigen Jahren wurde beim Schweinevirus ein Tropismenwechsel mit Beteiligung der Lunge beobachtet, der mit einer hohen Letalität einherging [5]. Wegen der großen Bedeutung für die Letalität bei Schweinen, Katzen und Hühnern wurden auch schon Impfstoffe entwickelt [6].

\section{Virusaufbau und Replikationsmechanismus}

Coronaviren erscheinen im Elektronenmikroskop als polymorphe, kugelförmige Partikel von 60-220 nm Durchmesser und zeigen häufig eine typische fragile Corona, deren Untereinheiten (Zacken der Krone) Peplomere genannt werden. Diese vermitteln die Anheftung an die Wirtszellen. Das Genom besteht aus einer sehr großen einzelsträngigen Plusstrang-RNA von ca. 30.000 Basen. Nach Einbringen der RNA in Zielzellen kann der ReplikaseEnzymkomplex direkt abgelesen werden. Die Minusstrang-RNA dient dann als Matrize für weitere genomische RNA. Vom Plusstrang werden auch mehrere unterschiedlich lange subgenomische Minusstrangkopien abgelesen, deren beide Enden durch einen Translokationsmechanismus mit den Enden des Gesamtgenoms identisch sind. An den Plusstrangkopien dieser Intermediate werden alle weite- ren viralen Proteine synthetisiert. Insgesamt werden 4 Strukturproteine (3 Glykoproteine: S, E, M und ein Kapsidprotein: N) und eine Reihe von Nichtstrukturproteinen abgelesen. Wie bei den Flaviviren, die ebenfalls nur eine einzige Plusstrang-RNA enthalten, sind infizierte Zellen auch nach Azetonfixierung noch infektiös (eigene Beobachtung). Möglicherweise erklärt sich hieraus teilweise die hohe Restinfektiosität von Coronaviren in verschiedenen Ausscheidungen [6].

\section{Severe Acute Respiratory Syndrome Coronavirus (SARS-CoV)}

Das Auftreten eines neuen humanpathogenen Coronavirus Ende 2002 in Südchina hat das Interesse der gesamten Weltöffentlichkeit auf sich gezogen. Die Ereignisse, die eine weltweite Verbreitung des Virus einleiteten, glichen denen, die bei einer Pestepidemie auf St. Hubert Anfang des 20. Jahrhunderts eine Rolle spielten, wobei allerdings statt der Schiffe heute eher Flugzeuge eine Rolle bei der Verbreitung spielen. Wie bei dieser Epidemie hat auch SARS Opfer bei Ärzten und Pflegepersonal gefunden [7].

\section{$>$ Die globale Ausbreitung der SARS-Infektion war wohl auf einen einzigen erkrankten Menschen zurückzuführen}

Der genaue Ablauf der Ausbreitung von SARS wurde bald nach dem Ende der Epidemie im Mai 2003 recherchiert. So 
hat das neue Coronavirus wahrscheinlich bereits im November 2002 den ersten Krankheitsfall in der Stadt Fushan in der Provinz Guangdong (Kanton) verursacht. Von dort wurde die Infektion oh-

Tabelle 1

Initiale Symptome bei $\mathbf{5 0}$ gesicherten SARS-Patienten aus Hongkong. (Nach [12])

\begin{tabular}{ll|}
\hline Symptome & Anzahl [\%] \\
\hline Fieber & $50(100)$ \\
\hline Husten & $31(62)$ \\
\hline Myalgien & $27(54)$ \\
\hline Heiserkeit & $10(20)$ \\
\hline
\end{tabular}

Tabelle 2

Verdachtsfälle (suspected), wahrscheinliche (probable) und laborbestätigte (laboratory confirmed) Fälle von SARS im Jahr 2003 in verschiedenen Ländern (Daten von den Internetseiten der Centers for Disease Control and Prevention (CDC) und der Weltgesundheitsorganisation (WHO) Homepage: http://www.who. int/csr/sars/country/en/country2003_08_15.pdf, http://www.cdc.gov/od/oc/ media/sars/cases/html und eigene Daten)

\begin{tabular}{llcl} 
Land & Verdachtsfälle & Wahrscheinliche Fälle & Laborbestätigte Fälle \\
\hline USA & 164 & 19 & 8 \\
\hline GB & 22 & 8 & 1 \\
\hline Frankreich & 394 & 5 & 4 \\
\hline Deutschland & 211 & 9 & 3 \\
\hline Kanada & $?$ & 251 & $?$ \\
\hline China & $?$ & 5.013 & $?$ \\
\hline
\end{tabular}

Tabelle 3

Teilnehmer der ersten WHO-Telefonkonferenz am 17.3.03. Aus Deutschland wurden später noch die Institute für Virologie in Frankfurt und Marburg eingeschlossen

\begin{tabular}{ll}
\hline USA & Centers for Disease Control and Prevention (CDC), Atlanta GA \\
\hline Großbritannien & $\begin{array}{l}\text { Regional Virus Laboratory, Belfast } \\
\text { Aberdeen Royal Infirmany, Aberdeen } \\
\text { PHLS Central Public Health Laboratory, London }\end{array}$ \\
\hline Hongkong & $\begin{array}{l}\text { Government Virus Unit, Kowloon, Hongkong } \\
\text { Prince of Wales Hospital, The Chinese University of Hong Kong } \\
\text { University Pathology Building, Queen Mary Hospital }\end{array}$ \\
\hline Singapur & Singapore General Hospital \\
\hline Niederlande & National Influenza Centre, Rotterdam \\
\hline Kanada & $\begin{array}{l}\text { Health Canada, Winnipeg } \\
\text { Health Canada, Ottawa }\end{array}$ \\
\hline Deutschland & Bernhard-Nocht-Institut (BNI), Hamburg \\
\hline Frankreich & Institut Pasteur, Paris \\
\hline Japan & National Institute of Infectious Disease, Tokio \\
\hline
\end{tabular}

eine größere Zahl von Personen aus Hongkong, weiter ein Ehepaar, das nach Toronto (Kanada) [10] zurückkehrte, einen Geschäftsmann, der nach Hanoi (Vietnam) reiste, sowie 3 Personen, die nach Singapur flogen. Schließlich waren zwischen 5.000 und 8.000 Personen weltweit infiziert. Der ganz überwiegende Teil stammte allerdings aus China. Erste Berichte an die Weltgesundheitsorganisation (WHO) über ein schweres akutes respiratorisches Syndrom (Severe Acute Respiratory Syndrome: SARS) als neue klinische Entität stammten von Dr. Carlo Urbani, der im März 2003 während seiner Arbeiten in Hanoi, Vietnam, den Begriff geprägt hat. Er selbst erkrankte Mitte März 2003 in Hanoi an SARS und verstarb am 29. März in Bangkok (Thailand).

\section{Klinisches Bild und Behandlung}

Wegen der häufigen Infektion des medizinischen Personals ließ sich schon bald eine kurze Inkubationszeit von 2 bis maximal 10 Tagen feststellen. Als erste Symptome werden Fieber, Muskel- und Kopfschmerzen angegeben. Respiratorische Beschwerden finden sich häufig erst am dritten bis fünften Krankheitstag, wobei Fieber, trockener Husten und Atemnot im Vordergrund stehen (• Tabelle 1). In schweren Fällen zeigt die Blutgasanalyse eine Hypoxie. Im Röntgenbild kommt es fortschreitend zu peripheren Infiltrationen. Auffallend oft ist eine starke Erhöhung der Laktatdehydrogenese (LDH) $[11,12]$. Man vermutet eine immunologische Komponente; jedenfalls hat sich die massive Gabe von Kortikosteroiden als hilfreich für die Behandlung von SARSPatienten herausgestellt [9]. Während bei jungen Patienten die Sterblichkeitsraten relativ gering waren $(2-5 \%)$, fand sich eine hohe Letalität (bis ca. 40\%) bei Menschen über 60 Jahren [13].

\section{Übertragung}

Schon zu Beginn der Epidemie fiel auf, das vor allem Krankenhauspersonal und Krankenhauspatienten (62\% aller Fälle in Hongkong, [14]) betroffen waren. Anfangs wurde offenbar die aerogene Ausbreitung der Erreger unterschätzt, und entsprechende Schutzmaßnahmen (Ge- 
sichtsmasken) wurden nicht konsequent verwendet. Obwohl das Virus in späteren Stadien der Erkrankung auch in großen Mengen im Stuhl vorkommt, sind fäkal-orale Übertragungen wohl selten, da die schwerkranken Patienten meist hospitalisiert sind und eine ausgeprägte Diarrhöe mit entsprechenden Kontaminationen nicht im Vordergrund steht. Die Hauptinfektionsgefahr geht also vom Respirationstrakt hospitalisierter Patienten aus. Die Schwerkranken im Krankenhaus sind offenbar besonders infektiös (z. B. viele Infektionen in Kanada bei Intubationen).

\section{(D) Die Hauptinfektionsgefahr geht vom Respirationstrakt hospitali- sierter Patienten aus}

Dies stimmt mit der Beobachtung überein, dass die Patienten in den ersten Krankheitstagen nur niedrige Viruskonzentrationen im Sputum aufweisen und im Gegensatz zu Influenzapatienten - erst am 5. Tag ein entsprechendes Maximum erreichen [12]. An dem letzten leicht verlaufenden Fall eines chinesischen Reporters im Dezember 2003 hatte sich von ungefähr 100 Kontaktpersonen niemand angesteckt [15], was zum Teil aber auch an der neuen Wachsamkeit der Bevölkerung liegen kann. Auch im Stuhl können die Viren in infektiöser Form über mehrere Wochen ausgeschieden werden. Schließlich kommen mit der weiten Verbreitung des Virus in Forschungs- und Diagnostiklaboratorien auch Laborinfektionen vor. Ein besonders hoher Aerosolschutz (z. B. Kopfmaske mit gefilterter Luftzufuhr) wäre daher wohl bei Arbeiten mit größeren Virusmengen anzuraten.

\section{Diagnostik und Epidemiologie}

Aufgrund der klinischen Daten wurden die Patienten in Verdachtsfälle (suspected) und wahrscheinliche Fälle (probable) eingeteilt [16]. Kriterien für diese Einteilung waren Aufenthalt in Endemiegebieten innerhalb der Inkubationszeit und klinisch schwere Respirationstraktsymptome einschließlich Röntgenbefund. Es ist verständlich, dass viele Menschen, die mit leichten Grippesymptomen aus Hongkong kamen, schon als suspected geführt wurden. Erst durch die Möglichkeit, klinische Daten mit einer virologischen Labordiagnostik zu untermauern, zog mehr Klarheit in die Diagnostik ein. Es gibt inzwischen eine neue Definition für die bestätigten Fälle (laboratory confirmed). Diese schließt die Labordiagnose, also einen positiven Virusnachweis üblicherweise mit der RT-PCR oder einen positiven Antikörpertest 4 Wochen nach Krankheitsbeginn mit ein. Antikörper werden vorzugsweise mit der indirekten Immunfluoreszenz nachgewiesen (• Abb.1). IgM-Antikörper sind nur kurze Zeit früher als die IgG-Antikörper nachweisbar [17]. Bei akut Erkrankten ist der erfolgreiche Nachweis mit sehr empfindlichen RT-PCR-Techniken fast zu 100\% möglich, wenn Auswurf am 3.-6. Krankheitstag untersucht wird. Hier können dann hohe virale RNA-Konzentationen von bis zu 100 Million Molekülen pro Milliliter gefunden werden. Weniger erfolgreich ist der Nachweis im Rachenabstrich. Eher selten wird Virus auch im Serum nachgewiesen [18]. Im Stuhl kommt es zu einer Ausscheidung nach dem 7 . Krankheitstag, die über 2 Wochen andauern kann [19]. Die Zahlen der laborbestätigten Fälle liegen insbesondere in den Nicht-Endemiegebieten dramatisch unter denen, die anfänglich aufgrund der klinischen Daten genannt wurden [16] (• Tabelle 2).

Daten über die serologisch bestätigten Fälle liegen für viele Regionen immer noch nicht vor, obwohl sie leicht über Antikörperteste zu erheben wären. So gibt es für China bislang keine genauen Daten darüber, wie viele der klinisch wahrscheinlichen Fälle am Jahresende 2003 wirklich Antikörper entwickelt haben. Natürlich sind auch alle Mortalitätsdaten unzuverlässig, wenn man nur Zahlen der klinisch wahrscheinlichen, nicht aber die der serologisch bestätigten Fälle kennt. Von den 38 in Europa gemeldeten wahrscheinlichen Fällen sind nach Labortestung auf SARS nur 8 antikörperpositive Personen übrig geblieben, von denen einer erst nach seiner Rückreise von Frankreich nach Vietnam erkrankte. Immerhin sind 552 Patienten weltweit an SARS gestorben, in Europa und den USA übrigens keiner. Man kann aber nicht sicher sein, ob wirklich alle verstorbenen Patienten eine Infektion mit SARS-Co-
Bundesgesundheitsbl - Gesundheitsforsch Gesundheitsschutz 2004 - 47:647-652 DOI 10.1007/s00103-004-0868-6 ๑) Springer-Verlag 2004

\section{H. Schmitz · C. Drosten}

\section{Die Bedeutung von Coronaviren. Das Beispiel SARS}

\section{Zusammenfassung}

Die Bedeutung der Coronavirus-Infektionen beim Menschen hat sich durch das Auftauchen des SARS-Coronavirus dramatisch erhöht. In dieser Übersicht werden sowohl die epidemiologischen wie auch die klinischen Aspekte dieser neuen Virusinfektion dargestellt. Ausführlich wird auch die Rolle der virologischen Forschung bezüglich Pathogenese, Diagnostik und Therapie abgehandelt. Die jüngsten Infektionen im Dezember 2003 zeigen, dass das Thema SARS uns auch im Jahr 2004 noch weiterhin beschäftigen wird.

\section{Schlüsselwörter}

Coronaviren· SARS

\section{Relevance of coronaviruses. The SARS example}

\begin{abstract}
The emergence of the new SARS coronavirus has shown dramatically the high relevance of human coronavirus infections. This overview presents data of both epidemiological and clinical studies of this new virus infection. Moreover, details of research on the pathogenesis, diagnostics, and therapy are given. The recent infections in December 2003 clearly document that SARS will be a major topic also in 2004.
\end{abstract}

\section{Keywords}

Coronaviruses · SARS 

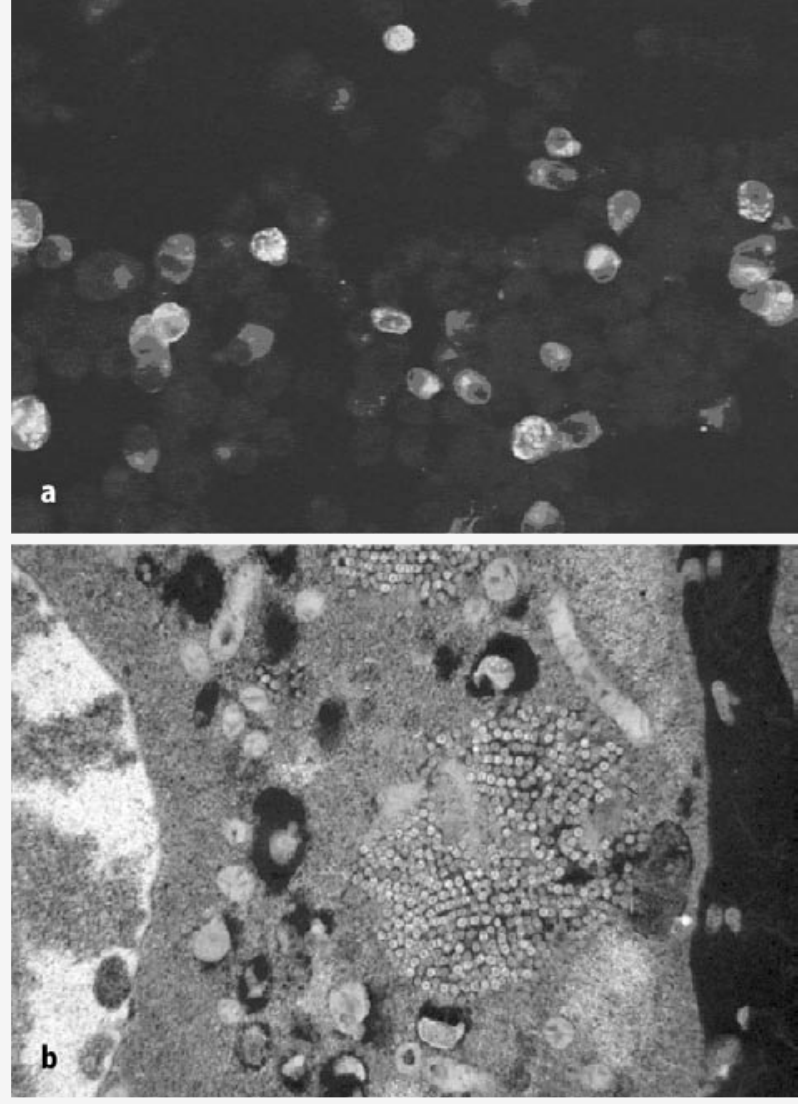

Abb. $1<$ a Indirekte Immunfluoreszenz von SARS-CoV-infizierten Verozellen. $b$ Parakristalline Ablagerung von Coronavirus Kapsiden in Verozellen (EM Vergr. 20.000:1)

\section{Gruppe 2}

\section{Gruppe 1}

Murine hepatitis virus

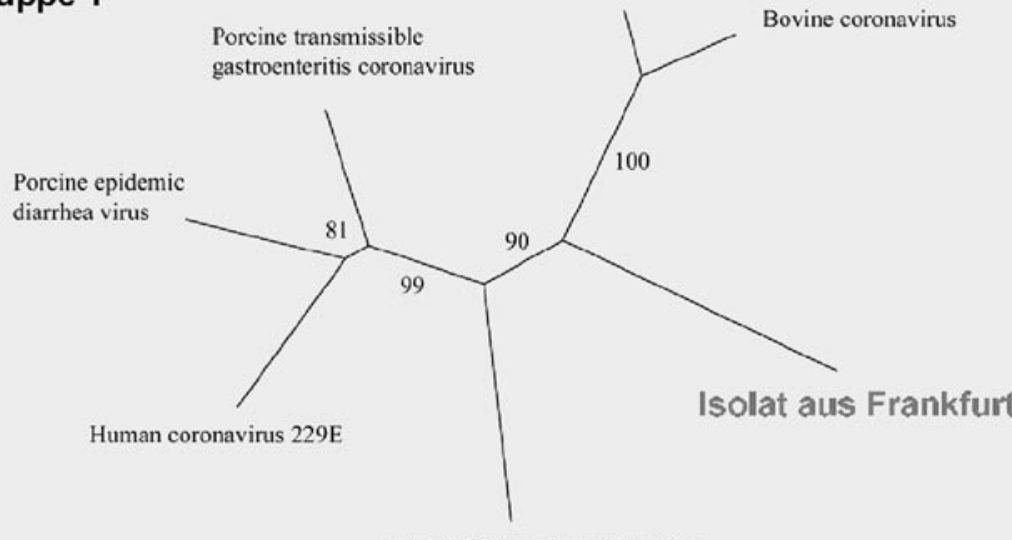

Avian infectious bronchitis virus

\section{Gruppe 3}

Abb.2 $\triangle$ Phylogenese der Coronaviren und Einteilung in Gruppen, basierend auf einem der ursprünglich gefundenen Fragmente des SARS-CoV Replikasekomplex-Gens [24]. Das Isolat aus Frankfurt stellt eine eigenständige Gruppe dar, die am nächsten mit Gruppe 2 verwandt ist

rornavirus aufgewiesen haben. Bei den wahrscheinlichen Fällen, die überlebten, muss die anfänglich genannte Zahl von ca. 8.000 aber sicherlich halbiert werden, wenn man die heutigen Kriterien anlegt. Die Identifizierung des Virus und die da- mit verbundene Möglichkeit einer virologischen Diagnostik werden also die Zahlen über das Ausmaß der Epidemie und über die Todesraten nachhaltig verändern. Immerhin lassen aber die Vergleiche von klinischen und serologischen $\mathrm{Da}$ - ten des Pflegepersonals aus Singapur vermuten, dass die meisten infizierten Personen auch schwer erkrankten [20].

\section{Identifizierung des Virus}

Als die Daten von Dr. Urbani aus Vietnam zur WHO gelangten, wurde durch diese eine Reihe von Laboratorien eingeschaltet, um den Krankheitserreger zu identifizieren. Da man aufgrund der klinischen Symptomatik von einem Influenza-ähnlichen Virus ausging und auch über Hinweise auf eine Influenzavirusinfektion bei Patienten mit SARS-ähnlichen Symptomen berichtet wurde, handelte es sich zunächst um Labore, die sich mit der Epidemiologie und Diagnostik von Influenzaviren beschäftigten. Schon Anfang März 2003 stellte sich jedoch heraus, dass eine Influenzavirusinfektion wegen der vielen negativen Befunde in den Blutund Sputumproben der Patienten ausgeschlossen werden konnte. Eine Virusinfektion lag aber wegen des Nicht-Anprechens auf Antibiotika nahe. Wie in $\bullet$ Tabelle 3 aufgelistet, wurden nun auch Laboratorien einbezogen, die sich mit der Diagnostik exotischer Virusinfektionen befassen. Diese Laboratorien wurden in einer ersten Telefonkonferenz der WHO zusammengeschaltet [16].

Am 15.3.03 wurde ein Arzt aus Singapur mit seiner Frau und dessen Schwiegermutter in das Universitätskrankenhaus in Frankfurt am Main eingeliefert. Mit großer Wahrscheinlichkeit handelte es sich um SARS-Patienten. Über das WHO-Netzwerk wurden in weiteren Konferenzen Ausschlussdiagnosen erstellt. In den umfangreichen Untersuchungen der Virologie des Bernhard-Nocht-Institutes konnten Infektionen mit hämorrhagischen Fieberviren und mit Flavivirus ausgeschlossen werden. Im Elektronenmikroskop wurden im Patientensputum Viruspartikel gefunden, die man zuerst für Parainfluenzaviren hielt [10]. Auch Chlamydien sollten eine Rolle spielen [21]. Allerdings konnten andere Laboratorien diese Daten nicht bestätigen [19]. In der Virologie in Frankfurt am Main gab es Hinweise darauf, dass sich in Gewebekulturen (Verozellen), die mit dem Patientenmaterial beimpft worden waren, ein zytopathischer Effekt einstellte. Der Ge- 
webekulturüberstand wurde nach Hamburg übermittelt und dort mit einer Random-Priming-Methode auf das Vorhandensein von viraler RNA untersucht. Dabei zeigte sich, dass 2 Sequenzen amplifiziert werden konnten, die eine geringe, aber doch eindeutige Homologie zum Replikase-Genkomplex von tierischen Coronaviren aufwiesen. Aufgrund der Sequenzdaten konnte es sich um ein neues, bis dahin unbekanntes Coronavirus handeln (• Abb.2) [19]. Zeitgleich wurden ähnliche PCR-Befunde auch von einer Gruppe in Hongkong erhoben [22], und Forscher der CDC photographierten Coronavirus-ähnliche Partikel im Elektronenmikroskop [23]. Mit den Primern, die in Hamburg an die Sequenz des neuen Virus angepasst und dann weltweit zur Verfügung gestellt wurden, zeichnete sich bald ab, dass bei praktisch allen akuten, schwer erkrankten Patienten in Hongkong und in Vietnam CoronavirusRNA vorhanden war [24]. In Immunfluoreszenztesten konnten auch bald bei Rekonvaleszenten Antikörper gegen das neue Coronavirus nachgewiesen werden (- Abb.1a). Schließlich kam es nach Infektion mit dem SARS-CoV auch in Makaken zu Erkrankungen, sodass ein Tiermodell entwickelt werden konnte [25].

\section{Sequenzanalysen}

Schon wenige Wochen nach Isolierung des SARS-CoV waren die 30.000 Basen des viralen Genoms sequenziert [26, 27, 28]. Diese Ergebnisse bestätigten die ersten Sequenzdaten, die bereits gezeigt hatten, dass es sich um ein neues Coronavirus handeln musste. Es wurde daher in eine vorläufige Gruppe 4 der Familie Coronaviridae eingeordnet ( $\bullet$ Abb. 2). Dem SARS-CoV fehlt im Vergleich zu anderen Coronaviren der Gruppe 2 ein Hämagglutininesterase-Gen sowie eine der beiden papainähnlichen Proteasen. Die Hauptprotease ist jedoch auch hier ein Chymotrypsin-ähnliches Enzym, das möglicherweise einen guten Angriffspunkt für eine antivirale Therapie darstellt [29,30]. Schließlich wurde auch das Gen für das Spike-Protein kloniert und der dazu passende Zellrezeptor identifiziert [31]. Bei der Analyse verschiedener SARS-CoVIsolate aus Menschen zeigten sich bislang
2 Genotypen, wobei bei allen Patienten, die in Verbindung mit den Ausbruch im Hotel M. in Hongkong standen, ein übereinstimmender Genotyp vorlag.

\section{Tierreservoir}

Schon die ersten serologischen Befunde zeigten, dass bei Deutschen, die nicht in Südostasien gewesen waren, keine Antikörper gegen SARS-CoV vorkommen. Daher war anzunehmen, dass das Virus nicht weltweit verbreitet sein konnte. Wegen des Vorkommens von anderen Coronaviren in Tieren wurde vermutet, dass bestimmte Tiere in der Guangdong-Provinz die eigentlichen Wirte des SARS-CoV sein könnten und dass das Virus nur gelegentlich auf den Menschen übertragen werden würde.

\section{SARS-CoV-ähnliche Sequenzen wurden in einer Reihe von Wildtieren gefunden}

Möglicherweise stand das Tier auf den vielfältigen Speisezetteln in Südchina. Dafür sprach auch ein im Vergleich zur Normalbevölkerung in Guangdong erhöhter Anteil von Nahrungshändlern und Küchenpersonal bei den SARS-Erkrankten in Guangdong. Nachdem eine PCR-Nachweismethode in China etabliert worden war, wurden natürlich die verschiedenen Schlachttiere auf den lokalen Märkten untersucht. SARS-CoV-ähnliche Sequenzen wurden in einer Reihe von Wildtieren gefunden, am häufigsten bei Larvenrollern (masked palm civet, Pagima larvata, Abb.3). Das Fleisch dieser Tiere wird in Südchina als Delikatesse verzehrt, deshalb werden die Tiere auch in Gehegen in großer Zahl gezüchtet. Ihre unnatürliche Haltung in Gefangenschaft wird sicher zur Ausbreitung der Infektion in diesen Tieren beitragen. Ende Dezember erkrankte ein 32-jähriger chinesischer Reporter relativ leicht an einer SARS-CoVInfektion. Die Sequenz seines Virusisolates war nahezu 100\%ig mit der Virussequenz, die in Larvenrollern gefunden wurde, identisch. Die Verwandtschaft zu Viren aus den im Frühjahr erkrankten Menschen war hingegen deutlich geringer. Dieser Befund untermauert die Hypothese eines zoonotischen Ursprunges des SARS-CoV. Allerdings hatte der

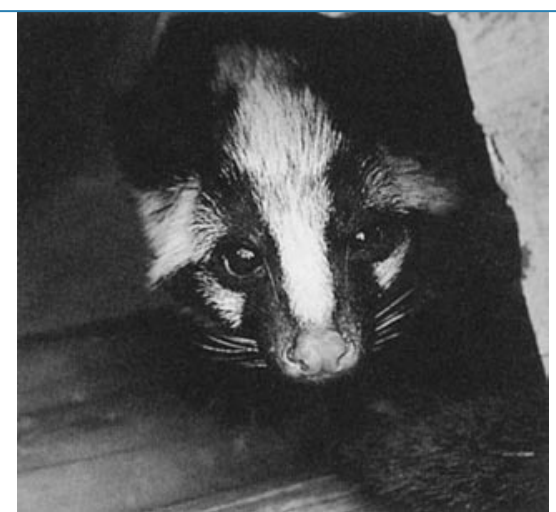

Abb. $3 \Delta$ Larvenroller (masked palm civet, Pagima larvata)

Reporter keine offensichtlichen Kontakte zu den Tieren, er hatte aber in seiner Wohnung Ratten gefangen [15]

Neben den beiden bisher berichteten Laborinfektionen (Singapur/Taiwan) treten also offenbar auch Neuinfektionen bei Menschen auf, die anderweitig mit dem Virus Kontakt hatten. Immer wieder wird auch über Infektionen von Tieren berichtet, wobei die Käfighaltung die Weitergabe von Tier zu Tier begünstigen dürfte. Es ist zu hoffen, dass der eigentliche Wirt des SARS-CoV, der das Virus auf Tiere wie den Larvenroller und auch auf Menschen überträgt, bald identifiziert wird. Ein sorgfältigerer Umgang mit dem aerosolgängigen, hochpathogenen SARS-Coronavirus wäre bei allen Laborarbeiten weltweit wünschenswert.

\section{Korrespondierender Autor \\ Prof. Dr. H.Schmitz}

Abteilung für Virologie,

Bernhard-Nocht-Institut für Tropenmedizin, Bernhard-Nocht-Straße 74, 20359 Hamburg

E-Mail:schmitz@bni-hamburg.de

\section{Literatur}

1. Tyrell D, Bynoe ML (1965) Cultivation of a novel type of commoncold virus in organ culotures. Br Med J 1:14671470

2. Monto AS, Lim SK (1974) The Tecumseh study of respiratory illness. VI. Frequency of and relationship between outbreaks of coronavirus infection. J Infect Dis 129:271-276

3. Pitkaranta A et al. (1998) Detection of rhinovirus, respiratory syncytial virus, and coronavirus infections in acute otitis media by reverse transcriptase polymerase chain reaction. Pediatrics 102 (2 Pt 1):291-295

4. Kern P et al. (1985) Detection of coronavirus-like particles in homosexual men with acquired immunodeficiency and related lymphadenopathy syndrome. Klin Wochenschr 63:68-72

5. Laude H, Van Reeth K, Pensaert M (1993) Porcine respiratory coronavirus: molecular features and virus-host interactions. Vet Res 24(2):125-150 
6. Holmes KV (2001) Coronaviruses. In: Fields B (ed) Virology. Lippincott, Raven, New York, pp 1055-1062

7. Lewis S (1998) Arrowsmith. Penguin Books ed. 1925, Harcourt Barce \& World, New York

8. Chan-Yeung M, Yu WC (2003) Outbreak of severe acute respiratory syndrome in Hong Kong Special Administrative Region: case report. Bmj 326:850-852

9. Zhao Z et al. (2003) Description and clinical treatment of an early outbreak of severe acute respiratory syndrome (SARS) in Guangzhou, PR China. J Med Microbiol 52 (Pt 8):715-720

10. Poutanen SM et al. (2003) Identification of severe acute respiratory syndrome in Canada. $\mathrm{N}$ Engl $\mathrm{J}$ Med 348:1995-2005

11. Hawkey PM, Bhagani S, Gillespie SH (2003) Severe acute respiratory syndrome (SARS): breath-taking progress. J Med Microbiol 52 (Pt 8):609-613

12. Peiris JS et al. (2003) Clinical progression and viral load in a community outbreak of coronavirus-associated SARS pneumonia: a prospective study. Lancet 361:1767-1772

13. Donnelly CA et al. (2003) Epidemiological determinants of spread of causal agent of severe acute respiratory syndrome in Hong Kong. Lancet 361:1761-1766

14. LeeN etal.(2003) A major outbreak of severe acute respiratory syndrome in Hong Kong. N Engl J Med 348:1986-1994

15. WHO (2004) Laboratory confirmation of a SARS case, southern China. Weekly Epidemiology Record, Genf, pp 1-12

16. WHO (2003) A multicentre collaboration to investigate the cause of severe acute respiratory syndrome. Lancet 361:1730-1733

17. Li G, Chen X, Xu A (2003) Profile of specific antibodies to the SARS-associated coronavirus. N Engl J Med 349:508-509

18. Zhai J et al. (2004) Real-time polymerase chain reaction for detecting SARS. Coronavirus, Beijing. Emerg Inf Dis 10:300-3003

19. Drosten C et al. (2003) Identification of a novel coronavirus in patients with severe acute respiratory syndrome. N Engl J Med 348:1967-1976

20. Ho KY et al. (2004) Mild illness associated with severe acute respiratory syndrome coronavirus infection: lessons from a prospective seroepidemiologic study of health-care workers in a teaching hospital in singapore.J Infect Dis 189:642-647

21. Enserink M (2003) SARS in China. China's missed chance. Science 301:294-296

22. Peiris JS et al. (2003) Coronavirus as a possible cause of severe acute respiratory syndrome. Lancet 361:1319-1325

23. Ksiazek TG et al. (2003) A novel coronavirus associated with severe acute respiratory syndrome. $\mathrm{N}$ Engl J Med 348:1953-1966

24. Drosten C et al. (2003) Severe acute respiratory syndrome: identification of the etiological agent. Trends $\mathrm{Mol}$ Med 9:325-327

25. Fouchier RA et al. (2003) Aetiology: Koch's postulates fulfilled for SARS virus. Nature 423:240

26. Ruan YJ et al. (2003) Comparative full-length genome sequence analysis of 14 SARS coronavirus isolates and common mutations associated with putative origins of infection. Lancet 361:1779-1785

27. Rota PA et al. (2003) Characterization of a novel coronavirus associated with severe acute respiratory syndrome. Science 300:1394-1399

28. Marra MA et al. (2003) The Genome sequence of the SARS-associated coronavirus. Science 300:1399-1404

29. Thiel V et al. (2003) Mechanisms and enzymes involved in SARS coronavirus genome expression. J Gen Virol 84 (Pt 9):2305-2315

30. Anand K et al. (2003) Coronavirus main proteinase ( $3 \mathrm{CL}$ pro) structure: basis for design of anti-SARS drugs. Science 300:1763-1767

31. Li W et al. (2003) Angiotensin-converting enzyme 2 is a functional receptor for the SARS coronavirus. Nature $426: 450-454$
A. Grohmann (Hrsg.)

\section{Karl Höll - Wasser, Nutzung im Kreislauf, Hygiene, Analyse und Bewertung}

Berlin, New York: Verlag de Gruyter 2002,

8. Auflage, 956 S., 105 Abb., 124 Tab.,

(ISBN-3-11-012931-0), 88.00 EUR

"Der Höll", seit nahezu 60 Jahren ein Standardwerk für alle, die sich mit dem Schutz des Trinkwassers befassen, ist in der 8. Auflage erschienen. Nach dem Tod von Karl Höll 1997 übernahm Andreas Grohmann, ehemaliger Abteilungsleiter Trinkwasser im Umweltbundesamt die Herausgeberschaft.

"Nach wie vor ist das Buch in erster Linie dem Trinkwasser gewidmet (....) Das Buch ist aus der Praxis für die Praxis leicht verständlich geschrieben, um allen Wasserfachleuten, Hygienikern, Technikern, Biologen und Chemikern u.a. die so notwendige Zusammenarbeit zu erleichtern", schreibt Höll 1970 im Vorwort zur 5. Auflage, und diese Aussage kann auch 30 Jahre später noch als gültig angesehen werden.

Seit der letzten Auflage aus dem Jahr 1986 hat sich in der Wasserwirtschaft vieles getan, das in der Neuauflage berücksichtigt werden musste. So ist zu begrüßen, dass in Zeiten zunehmender Belastungsrisiken für die Ressource Wasser ein Kapitel über die hydrogeologischen Grundlagen aufgenommen wurde. Weiter haben sich die Möglichkeiten und Notwendigkeiten der chemischen und mikrobiologischen Wasseranalyse entwickelt, neue Verfahren, z.B. Immunoassays sind auf den Markt gekommen. Die Zahl der relevanten chemischen und mikrobiologischen Noxen wird größer. Fortzuschreiben waren auch vor allem die Kapitel, die Vorkommen und Wirkungsweise dieser Noxen beschreiben. Dies war vor allem nach der Novelle der Trinkwasserverordnung 2001 erforderlich.

Die Veränderungen und Fortschritte im Bereich Wassergewinnung, -aufbereitung und -untersuchung sind jedoch nicht allein für die nahezu Verdoppelung des Buchumfangs auf 950 Seiten verantwortlich. Bereits der alte "Hölll" wollte Trinkwasser nicht als isoliertes Thema, sondern im Zusammenhang mit Betriebswasser, Badewasser, Flusswasser und Abwasser sehen. Diesem Anspruch fühlt sich auch Grohmann verpflichtet. Er öffnet den Blickwinkel auf nahezu alle wasserwirtschaftlichen Themen. Die Eutrophierung von Seen und ihre Sanierung ist ebenso ein Thema wie die biologische Gewässerklassifikation der
LAWA, die Abwasserreinigung oder die neue Wasserrahmenrichtlinie der EG, wenn auch der Bezug zum Trinkwasser nicht immer klar wird. Anzuerkennen ist auch der Versuch des Herausgebers, neue wasserpolitische Themen, wie z.B. die Liberalisierung und Privatisierung der Wasserversorgung oder die Regenwassernutzung, zu behandeln.

Welche Themen konkret aufgegriffen werden und die Vollständigkeit, in der sie behandelt werden, scheint jedoch stark durch die verantwortlichen Koautoren bestimmt worden zu sein. Nur so erklärt sich, dass die Auswahl zum Teil eine gewisse Beliebigkeit widerspiegelt. So sind z.B. für das Kapitel, das die Biotests behandelt - mit dem etwas unglücklich gewählten Titel „Toxikologie“ - , drei verschiedene Koautoren verantwortlich, die unabhängig voneinander diverse etablierte und - zum Teil aus gutem Grund - nicht etablierte Tests beschreiben, ohne deren Spezifik und Zweckgebundenheit deutlich zu machen.

Für den Insider der Wasserwirtschaft ist die Heterogenität des Buches kein größeres Problem, denn aufgrund des guten Stichwortregisters findet er (fast) alles, wenn auch zum Teil an unerwartetem Ort. Für den, der Orientierung sucht, wie z.B.Studenten, fehlt jedoch eine durchschaubare Systematik und die Hilfestellung, wesentliches von unwesentlichem zu unterscheiden. Es ist verständlich, dass bei einem über 60 Jahre sukzessive anwachsenden und sich erweiternden Buch rund um das Thema Trinkwasser die Gefahr besteht, dass der rote Faden verloren geht. Aber gerade wenn der "Höll“ auch in Zukunft ein Standardwerk bleiben soll, sollte sich der Herausgeber bei der nächsten Auflage zu einem stärkeren Eingreifen entschließen.

Christiane Markard (Berlin) 